\title{
Distinctive encoding reduces the Jacoby-Whitehouse illusion
}

\author{
David A. Gallo \\ University of Chicago, Chicago, Illinois \\ David H. Perlmutter \\ Harvard University, Cambridge, Massachusetts \\ Christopher D. Moore \\ Princeton University, Princeton, New Jersey \\ AND \\ DaNiel L. Schacter \\ Harvard University, Cambridge, Massachusetts
}

\begin{abstract}
We investigated the influence of distinctive encoding on the Jacoby and Whitehouse (1989) illusion. Subjects studied visually presented words that were associated with either an auditory presentation of the same word (nondistinctive encoding) or a picture of the object (distinctive encoding). In both conditions, words were visually presented on the recognition test, and half were preceded by brief repetition primes. Priming test items increased hits and false alarms in the auditory condition, demonstrating the Jacoby-Whitehouse illusion. This illusion was reduced in the picture condition. In order to test whether this distinctiveness effect was caused by a recollectionbased response strategy (i.e., the distinctiveness heuristic), we minimized recollection-based responding by having subjects make speeded recognition decisions. Contrary to the distinctiveness heuristic hypothesis, speeded responding did not eliminate the distinctiveness effect on the Jacoby-Whitehouse illusion. Picture encoding may reduce this illusion via a shift in preretrieval orientation, as opposed to a postretrieval editing process.
\end{abstract}

Recollection-based expectations are a fundamental aspect of retrieval monitoring, and research indicates that such expectations can influence a variety of memory biases and distortions (see, e.g., Johnson, Hashtroudi, \& Lindsay, 1993; Kelley \& Rhodes, 2002). For instance, using the DRM false memory task, Schacter, Israel, and Racine (1999) had subjects study lists of associates (e.g., door, screen) to nonstudied words (e.g., window). Each auditorily presented word was paired with the same word that was presented visually or with a picture, under the assumption that subjects would expect more distinctive recollections from pictures (i.e., more perceptually detailed and unique representations). False recognition of nonstudied associates was lower in the picture condition than in the visual-word condition, suggesting that subjects in the picture condition had used a distinctiveness heuristic. According to this hypothesis, nonstudied lures were unlikely to elicit distinctive picture recollections, and subjects inferred that the absence of such recollections was diagnostic of nonoccurrence. By contrast, after studying visual words, subjects were more likely to base their decisions on feelings of familiarity, leading to greater false recognition. Several recent studies suggest that subjects can use the distinctiveness heuristic to enhance memory accuracy across a variety of tasks and situations (for reviews, see Gallo, 2006; Schacter \& Wiseman, 2006).

In the present study, we investigated whether the distinctiveness heuristic could reduce the Jacoby and Whitehouse (1989) illusion. In this illusion, briefly flashing a repetition prime (e.g., garden) just prior to a recognition test item (garden) increases the likelihood that subjects will respond "old" to the test item, in comparison with a mismatched control prime (e.g., cookie). This priming effect is found for both targets and lures. Jacoby and Whitehouse claimed that the prime had to be processed unconsciously in order to influence the recognition decision, although subsequent findings indicate that conscious processing of the prime can also give rise to the illusion, as long as the primes are subtle (see Bernstein \& Welch, 1991; Gellatly, Banton, \& Woods, 1995; Higham \& Vokey, $2000)$. Either way, there is converging evidence supporting Jacoby and Whitehouse's conclusion that these sorts of manipulations enhance the processing fluency of the test item, yielding a subjective sense of familiarity rather than recollection (see, e.g., Rajaram, 1993; Westerman, 2001; Whittlesea, 1993). 
If the Jacoby-Whitehouse illusion is caused by familiarity-based attributions occurring at retrieval, then it should be eliminated by the use of a recollection-based distinctiveness heuristic. Westerman, Miller, and Lloyd (2003) reported results that were consistent with this hypothesis. They found that the Jacoby-Whitehouse illusion was eliminated in a condition in which subjects studied pictures in comparison with visual words (always using visual words at test). However, they argued that the illusion was eliminated because of a change in presentation format between study and test (i.e., a mismatch hypothesis) — not because subjects relied more on distinctive recollections at test in the picture condition (i.e., the distinctiveness heuristic hypothesis). According to the mismatch hypothesis, subjects in the picture condition did not expect test primes to be a useful source of information for their recognition decisions, because a different presentation format was used between study and test. Ostensibly, subjects assumed that prime-induced familiarity was diagnostic of study-phase presentation only when study and test presentation formats were identical (as is the case in typical Jacoby and Whitehouse procedure). As a further test of this mismatch hypothesis, Westerman et al. (2003) had subjects study a "counterfeit" study list (visual noise without studied items), which led them to believe that they had been subliminally presented with pictures. Even though these subjects could not recollect distinctive pictures and thus could not have used a distinctiveness heuristic, the Jacoby-Whitehouse illusion still was eliminated. The illusion was eliminated simply because subjects thought that the study and test formats were mismatched, not because one format was more distinctive than the other.

We agree with Westerman et al. (2003) that the counterfeit list results cannot be attributed to a recollection-based process, but because subjects in this condition were never presented with a study list, the generalization of these results to the recognition-memory situation is questionable. In particular, these results do not exclude the possibility that a distinctiveness heuristic was used to suppress the illusion when subjects actually had studied pictures and thus could have expected more distinctive recollections. The present task provided another test of the distinctiveness heuristic hypothesis. One critical methodological difference from Westerman et al. (2003) is that we used a visual presentation of words at both study and test in all conditions. Recollective distinctiveness was manipulated at study by associating each visual word with either an auditory presentation of this same word (the auditory condition) or a picture of the corresponding object (the picture condition). The distinctiveness heuristic hypothesis predicts that the Jacoby-Whitehouse illusion will be reduced in the picture condition, in comparison with the auditory condition. It also predicts that unprimed false recognition will be reduced in the picture condition, in comparison with the auditory condition (cf. Gallo, Weiss, \& Schacter, 2004; Schacter et al., 1999), thereby providing an independent measure of the distinctiveness heuristic.

In contrast, an account based on study-to-test format mismatches does not necessarily predict differences in the Jacoby-Whitehouse illusion between these conditions.
Each condition used the same visual-word presentation at study and at test. Further, each condition contained a study format that was different from the test format (auditory words or pictures). Westerman, Lloyd, and Miller (2002) reported that a study-to-test modality shift (i.e., auditory study-visual test) minimized the Jacoby-Whitehouse illusion, much like the picture condition of Westerman et al. (2003). If study-to-test mismatches were responsible for prior effects and recollective distinctiveness played no role, then these results suggest that using auditory presentation at study should have similar effects as would using picture presentation at study in our task. Because the objective match between study and test formats was held constant in the two study conditions of the present study (one matching format that has been found to elicit the Jacoby-Whitehouse illusion and one mismatching format that has been found to minimize the illusion), subjects in each condition should be equally susceptible to the Jacoby-Whitehouse illusion.

As an additional test of the distinctiveness heuristic hypothesis, we manipulated the speed with which subjects made their recognition decisions (self-paced or speeded). Dodson and Hege (2005) proposed that the distinctiveness heuristic is a time-consuming process, because it only applies after subjects attempt (and fail) to retrieve the expected recollections. Consistent with this prediction, they found that distinctiveness effects on DRM false recognition were eliminated when subjects were forced to respond within $750 \mathrm{msec}$. If the distinctiveness heuristic operates in the same way in the Jacoby-Whitehouse task, then speeding responses should eliminate distinctiveness effects on false recognition. Unlike the self-paced condition, false recognition of unprimed lures should not differ across the auditory and picture conditions under speeded conditions. Further, the Jacoby-Whitehouse illusion should be obtained in both the auditory and picture conditions under speeded conditions.

\section{METHOD}

\section{Subjects}

Ninety-six students recruited at Harvard University (mean age $=$ 19.9 years, $S D=2.8)$ participated for course credit or $\$ 10(n=$ 24 per condition). Data from one subject were replaced because of failure to follow instructions.

\section{Materials}

The study-phase stimuli were 176 words (four to eight letters) and corresponding line drawings (pictures) drawn from the Snodgrass and Vanderwart (1980) norms. These items were divided into four sets, equated on average word length. For counterbalancing, the items in each set appeared equally often (across subjects) in each of the four item-type conditions (i.e., studied status, target vs. lure; prime type, match vs. mismatch). For mismatch primes, each of the 176 study stimuli was paired with a high imagery word of identical length, so that the first letter of the two words differed. The two words were not obviously semantically related (e.g., swan-roof; suitcase-lemonade).

Each subject studied 88 items, with the remaining 88 serving as nonstudied lures on the recognition test. The first 16 test items were presented to subjects as practice (4 in each item-type condition), and the remaining 160 were presented as the main test (40 in each item-type condition). The order of study and test items was random- 
ized for each subject. All stimuli were presented using a G4 Power Macintosh and PsyScope OS 9 software.

\section{Procedure}

Subjects were tested individually. They were instructed that they would study a list of items for a subsequent memory test. In all conditions, each study word first was visually presented for $750 \mathrm{msec}$ (55-point font, lowercase, black on a white background). In the auditory condition, each visual word was followed by the same word spoken in a male voice (over the computer speakers); then, a blank screen filled the remaining $2 \mathrm{sec}$ prior to the next stimulus. In the picture condition, each visual word was followed by a corresponding picture for $2 \mathrm{sec}$ (black line drawing on a white background). Subjects in both the auditory and the picture conditions were told to focus on the visually presented words for the upcoming memory test and that the spoken words (or pictures) would help to provide additional cues.

Immediately following the study phase, the experimenter read the test instructions. All subjects were told that they would be presented with studied and nonstudied words and that they were to press the key labeled "yes" if they remembered studying the word, and the key labeled "no" if they did not. All test words and primes were presented in the same font as that used in the study phase. Each test word was preceded by a brief prime word $(40 \mathrm{msec})$, with half of the primes matching the test word, and the other half mismatching. Each prime was preceded and followed by a 200 -msec visual mask (\&\#\&\#\&\#\&\#\&) that covered the entire stimulus (these durations are analogous to those used by Westerman et al., 2003). Through pilot testing, we found that many subjects noticed that prime words had been presented; thus, we controlled this variable by telling all subjects about the presence of the primes. They were told that the mask would "randomly contain cue words" and that "although they will be very difficult to see, we believe that these cue words will sometimes help to cue, or automatically prime, your memory." These instructions established the same demand characteristics in all subjects and, in order to ensure that they focused on memory accuracy, all subjects were told "Be sure to only press 'yes' if you remember that the word was presented in the study list."

In order to control response speed, we adopted the recognitiontempo procedure (Balota, Burgess, Cortese, \& Adams, 2002). Subjects initiated each test trial with the space bar. In the speeded condition, they were presented with three simultaneous auditory beats and visual fixation cues, one every $650 \mathrm{msec}$. At the start of the second beat, the test prime was presented (200-msec premask, 40-msec prime, 200-msec postmask, 210-msec blank screen), and the test word was presented on the third beat. The test word remained on the screen until a response was made. Subjects were instructed to respond in time with the beat (i.e., around $650 \mathrm{msec}$ after the onset of the test word), and they were given a $250-\mathrm{msec}$ response window (500 msec-750 msec) during which a button press elicited the visual prompt GOOD! If they responded prior to the window, then they were prompted TOO FAST!, and if they responded after the window, then they were prompted TOO SLOW. In order to encourage responding within the window, the error prompts were accompanied by an auditory error message ("Uh-Oh"), and they remained on the screen for $3 \mathrm{sec}$. The self-paced condition was similar, but subjects were instructed to take their time to respond. A slower tempo of $900 \mathrm{msec}$ was established, and subjects were told to respond no faster than the tempo (in fact, most subjects took at least $1,500 \mathrm{msec}$ to respond, on average). If they responded within $750 \mathrm{msec}$, then they were prompted TOO FAST! If they responded after $750 \mathrm{msec}$, then they were prompted to press the space bar for the next trial. The same prime duration was used as that in the speeded condition (e.g., 200-msec premask, 40-msec prime, 200-msec postmask, 460-msec blank screen). We also tested subjects in an intermediate speed condition (750-msec-1,000-msec response window), but the results were similar to the self-paced condition and are thus not reported.

After the practice test, the experimenter started the main test. Following the main test, subjects were given a prime-identification test to ensure that the response-speed manipulation did not affect perceptual discrimination. This test used presentation procedures identical to those in the recognition test, but it contained 52 new items (half with matching primes, the other half mismatching). Subjects were instructed to press "yes" if they thought the prime matched the test word and "no" if they did not, using the same time constraints as those in the main test. Overall, subjects were more likely to respond "match" to matching primes (mean hits $=.85$ ) than to mismatched primes (mean FAs $=.07$ ), and there were no significant differences across the experimental conditions.

\section{RESULTS}

Recognition results for the self-paced test are presented in Figure 1. Unless otherwise noted, all results were significant $(p<.05$, two-tailed). A 2 (studied status: target vs. lure) $\times 2$ (prime type: match vs. mismatch) $\times 2$ (study format: auditory vs. picture) ANOVA revealed an effect of prime type $\left[F(1,46)=26.76, M S_{\mathrm{e}}=.007\right]$-demonstrating the Jacoby-Whitehouse illusion-and an interaction between prime type and study format $[F(1,46)=7.94$, $\left.M S_{\mathrm{e}}=.007\right]$. The interaction indicates that the illusion was greater in the auditory condition ( $10 \%$ across targets and lures) than in the picture condition (3\%). This reduction is similar to that reported by Westerman et al. (2003), and it indicates that objective study-to-test mismatches are not necessary to obtain this reduction. This reduction is more consistent with the distinctiveness heuristic hypothesis, although that conclusion should be withheld until the speeded results are presented. There was also an effect of studied status $\left[F(1,46)=422.77, M S_{\mathrm{e}}=.028\right]$, an effect of study format $\left[F(1,46)=6.55, M S_{\mathrm{e}}=.038\right]$, and an interaction between the two $\left[F(1,46)=12.65, M S_{\mathrm{e}}=\right.$ .028]. The interaction indicates that study format affected false alarms more than hits, which is consistent with the idea that the distinctiveness heuristic reduced false recognition without necessarily affecting true recognition (see, e.g., Gallo et al., 2004; Schacter et al., 1999). This finding is most easily seen in unprimed test items (i.e., those preceded by mismatching primes), which were unaffected

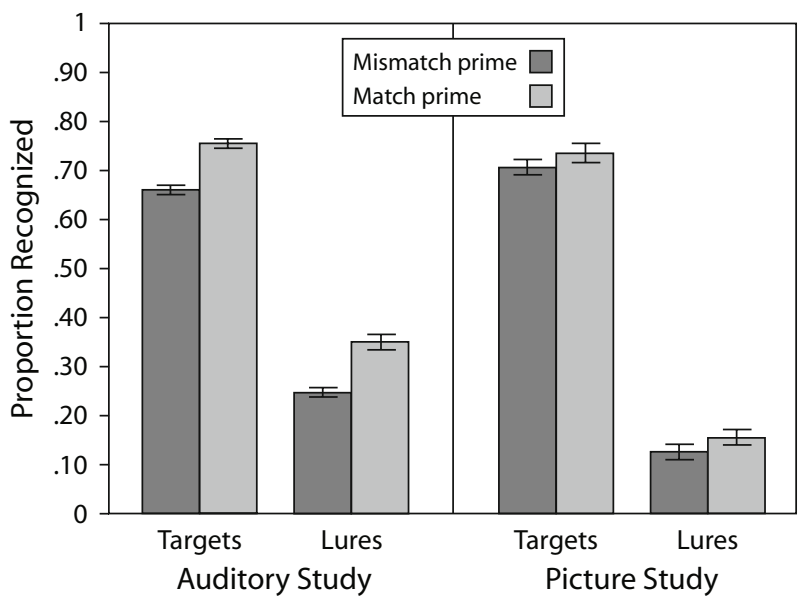

Figure 1.True and false recognition rates for the self-paced test, as a function of study format. Error bars represent standard errors of each mean. 
by prime-induced familiarity. Hits to unprimed targets did not differ across the study format conditions (auditory $=$ .66 , picture $=.71)[t(46)=1.24$, SEM $=.037, p=.22]$, but false alarms to unprimed lures were lower in the picture condition (.25 vs. .13) $[t(46)=3.13, S E M=.039$, $p<.01]$. No other effects or interactions were significant (all $F \mathrm{~s}<1$ ).

Speeded subjects were very good at responding within the 500-750-msec response window (average latency for all correct responses $=695 \mathrm{msec}, S E M=10 \mathrm{msec}$ ), and they were faster than subjects in the self-paced condition $(1,631 \mathrm{msec}, S E M=73 \mathrm{msec})[t(94)=12.62, S E M=$ 74.12]. On average, speeded subjects responded within the window on $73 \%$ of the trials in the auditory condition and on $58 \%$ of the trials in the picture condition, with most misses being too slow. A 2 (studied status) $\times 2$ (prime type) $\times 2$ (study format) ANOVA on the number of ontime trials indicated that this difference was significant $\left[F(1,46)=8.60, M S_{\mathrm{e}}=191.26\right]$, and there were no other main effects or interactions (all $F \mathrm{~s}<1$ ). Subjects may have taken longer to respond in the picture condition because they were more likely to use recollection in this condition than they were in the auditory condition. In fact, a similar (albeit nonsignificant) difference was observed on correct latencies on the self-paced tests $(M \mathrm{~s}=1,563 \mathrm{msec}$ for the auditory condition and 1,699 $\mathrm{msec}$ for the picture condition). Regardless of the cause of these differences, we analyzed the speeded recognition data both with and without responses outside the response window, and the results were very similar (maximum discrepancy in recognition means $=3 \%$ ). In order to avoid item selection confounds and maximize statistical power, we report data and analyses from all trials below, regardless of whether subjects responded on time. Unless otherwise noted, statistical results were identical for the two sets of analyses.

Recognition results from the speeded test are presented in Figure 2. Comparing these results with the self-paced results, a 2 (response speed) $\times 2$ (studied status) $\times 2$ (prime type) $\times 2$ (study format) ANOVA revealed three significant interactions. First, as was expected, studied status interacted with response speed $[F(1,92)=30.09$, $\left.M S_{\mathrm{e}}=.031\right]$. Collapsing across prime type and study format, speeded responding decreased hit rates (selfpaced hits $=.72$, speeded hits $=.63)[t(94)=3.02$, $S E M=.027]$ and increased false alarms (.22 and .34) $[t(94)=3.41, S E M=.034]$. There also was an interaction between study format and studied status $[F(1,92)=$ $\left.10.74, M S_{\mathrm{e}}=.031\right]$ and between study format and prime type $\left[F(1,92)=10.81, M S_{\mathrm{e}}=.011\right]$. As was discussed above, study format affected false recognition (auditory study $>$ picture study) more than true recognition, and it also affected the magnitude of the Jacoby-Whitehouse illusion (auditory study $>$ picture study). There were no other significant interactions, suggesting that the overall effects of study format were similar across the two response-speed conditions.

As in the self-paced condition, we conducted a 2 (studied status) $\times 2$ (prime type) $\times 2$ (study format) ANOVA on the speeded-condition data. There was an effect of prime type $\left[F(1,46)=18.94, M S_{\mathrm{e}}=.015\right]$ and an interaction

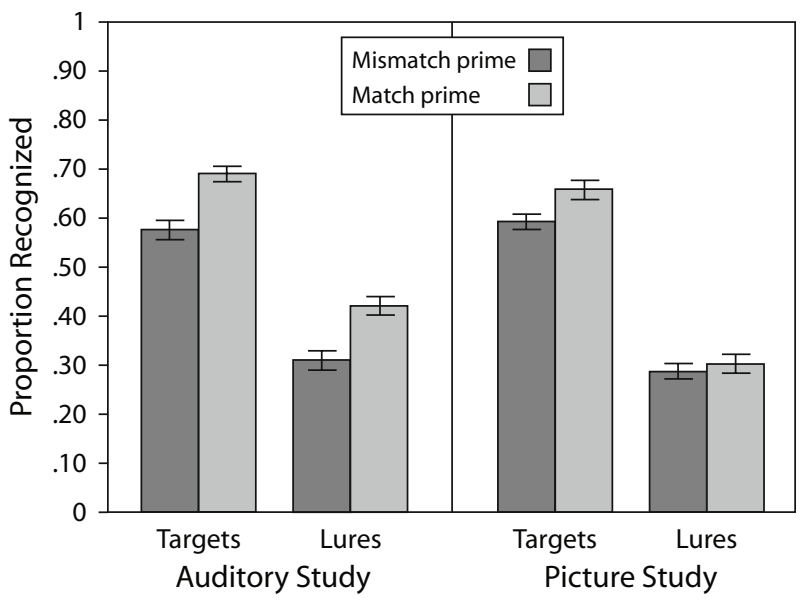

Figure 2. True and false recognition rates for the speeded test, as a function of study format. Error bars represent standard errors of each mean.

between prime type and study format $[F(1,46)=4.14$, $\left.M S_{\mathrm{e}}=.061\right] .{ }^{1}$ The interaction indicates that the JacobyWhitehouse illusion was again greater in the auditory condition (11\% across targets and lures) than in the picture condition $(4 \%) .^{2}$ There also was an effect of studied status $\left[F(1,46)=124.65, M S_{\mathrm{e}}=.034\right]$; however, unlike in the self-paced condition, there was no effect of study format $\left[F(1,46)=1.07, M S_{\mathrm{e}}=.067, p=.31\right]$ and no interaction between the two $\left[F(1,46)=1.45, M S_{\mathrm{e}}=.034, p=.24\right]$. Hits to unprimed targets did not differ across the study format conditions (auditory $=.58$, picture $=.60)[t(46)<1]$, and neither did false alarms to unprimed lures (.32 vs. .29) $[t(46)<1]$. This last comparison stands in contrast to the significant effect found on the self-paced test and suggests that speeded responding eliminated the distinctiveness heuristic on unprimed false recognition. Critically, however, the effect of distinctive encoding on the JacobyWhitehouse illusion persisted under speeded responding (auditory condition $>$ picture condition). This finding is inconsistent with the distinctiveness heuristic hypothesis.

As a final analysis, we compared overall levels of recognition discrimination $\left(d^{\prime}\right)$ across conditions. In the selfpaced condition, discrimination between targets and lures (collapsing across prime type) was greater in the picture condition (1.93) than in the auditory condition (1.12) $[t(46)=4.73, S E M=.170]$. This difference raises the possibility that the reduction of the Jacoby-Whitehouse illusion in the picture condition was due to overall levels of memory strength (and corresponding familiarity-based criterion shifts) as opposed to distinctiveness. However, this idea cannot explain why the Jacoby-Whitehouse illusion was minimized in the speeded-picture condition. In that condition, discrimination (.95) was no greater than it was in the self-paced auditory condition (1.12) $[t(46)=1.27, p=.21]$, in which a robust illusion was observed. Overall levels of memory strength (as estimated by $d^{\prime}$ ) cannot explain differences in the magnitude of the Jacoby-Whitehouse illusion that were observed across these conditions. 


\section{DISCUSSION}

Studying pictures reduced the Jacoby-Whitehouse illusion for both targets and lures, as compared with the auditory presentation condition. These effects replicate and extend those of Westerman et al. (2003), but importantly, they cannot be attributed to an objective format change between study and test. We presented visual words at study and at test in each condition, and through explicit instructions, we gave all subjects the same reason to believe that test primes might benefit their decisions. Despite these controls, subjects in the picture condition were less likely than those in the auditory condition to be influenced by the primes. By design, the major difference between conditions was in the perceptual distinctiveness of the secondary stimuli (pictures $>$ auditory words).

Some of our findings were predicted by the distinctiveness heuristic hypothesis, but others were not. Consistent with the use of a recollection-based distinctiveness heuristic, false recognition of unprimed lures was lower in the picture condition than in the auditory condition when responses were self-paced. Further, this effect was eliminated when responses were speeded. This last effect is a conceptual replication of Dodson and Hege's (2005) results in the DRM task, and it suggests that the distinctiveness heuristic was most likely to reduce false recognition to unprimed lures when subjects were given sufficient time to decide that they could not recollect a picture. These findings are consistent with a postretrieval monitoring interpretation of the distinctiveness heuristic, in which case subjects reject nonstudied lures only after a failed attempt to retrieve picture recollections. In general, these results add to the growing body of research indicating that expecting distinctive recollections can reduce false recognition across a variety of memory tasks.

Even though the distinctiveness heuristic hypothesis predicted the pattern of false alarms to unprimed lures, it did not predict the entire pattern of findings for the Jacoby-Whitehouse illusion. Specifically, picture encoding reduced this illusion in comparison with auditory encoding under both self-paced and speeded conditions. Although the distinctiveness heuristic could have minimized this illusion under the self-paced conditions, the speeded results are inconsistent with a distinctiveness heuristic explanation, because speeded responding should have minimized this recollection-based monitoring process. The most parsimonious explanation of these effects is that distinctive encoding reduced the Jacoby-Whitehouse illusion through some process other than a recollection-based monitoring process like the distinctiveness heuristic.

A modified interpretation of the study-to-test mismatch hypothesis that was originally proposed by Westerman and colleagues (e.g., Westerman et al., 2003) provides a more viable explanation of the current findings. Even though the objective match between study and test was held constant, one could argue that studying pictures nevertheless led to a greater subjective mismatch between study and test than did studying auditory words. Because pictures were more perceptually distinctive than auditory words, their salience might have dominated the encoding phase, thereby minimizing attention devoted to the visual words at study under both self-paced and speeded conditions. As a result, subjects may have believed that the perceptual mismatch between study and test was greater in the picture condition than in the auditory condition, causing subjects to discount perceptual fluency from the primes as a basis for responding. Unlike a postretrieval editing process, which would occur on a trial-by-trial basis, ignoring the test primes can be considered a preretrieval orientation process adopted for the entire test (cf. Jacoby, Kelley, \& McElree, 1999). The test-based nature of the Jacoby-Whitehouse illusion might make it particularly sensitive to such a preretrieval orientation, allowing subjects to avoid prime-induced familiarity by assuming that the primes were not diagnostic of study presentation.

In conclusion, our results demonstrate that prime words are less likely to influence recognition judgments when the studied materials are pictures. These results extend the work of Westerman and colleagues (Lloyd, Westerman, \& Miller, 2003; Westerman et al., 2003), providing further evidence that picture encoding can minimize the JacobyWhitehouse illusion even when subjects are unlikely to use recollection-based responding at test. Apparently, perceptual distinctiveness can suppress false recognition in more than one way. In some cases, distinctiveness appears to operate via a postretrieval editing process, such as the distinctiveness heuristic that potentially reduced unprimed false alarms in the present task and in others. In other cases, such as the Jacoby-Whitehouse illusion, distinctiveness can operate through a preretrieval orientation process that causes subjects to summarily discount potential sources of familiarity. In these cases, the critical factor is not so much that pictures elicit distinctive recollections, but that picture presentation is subjectively perceived as being qualitatively different from the verbal presentation used to prime memory at test. More generally, these findings suggest that the subjectively perceived overlap between encoding and retrieval conditions can influence retrieval monitoring, independent from recollection-based processes.

\section{AUTHOR NOTE}

This research was supported by National Institute on Aging Grants AG021369 to D.A.G. and AG08441 to D.L.S. Preliminary analyses of these data were presented at the 44th Annual Meeting of the Psychonomic Society in 2003 (Vancouver, BC). Address correspondence to D. A. Gallo, Department of Psychology, University of Chicago, 5848 S. University Ave, Chicago, IL 60637 (e-mail: dgallo@uchicago.edu).

\section{REFERENCES}

Balota, D. A., Burgess, G. C., Cortese, M. J., \& Adams, D. R. (2002). The word-frequency mirror effect in young, old, and early-stage Alzheimer's disease: Evidence for two processes in episodic recognition performance. Journal of Memory \& Language, 46, 199-226.

Bernstein, I. H., \& Welch, K. R. (1991). Awareness, false recognition, and the Jacoby-Whitehouse effect. Journal of Experimental Psychology: General, 120, 324-328.

Dodson, C. S., \& Hege, A. C. G. (2005). Speeded retrieval abolishes the false-memory suppression effect: Evidence for the distinctiveness heuristic. Psychonomic Bulletin \& Review, 12, 726-731.

Gallo, D. A. (2006). Associative illusions of memory: False memory research in DRM and related tasks. New York: Psychology Press. 
Gallo, D. A., Weiss, J. A., \& Schacter, D. L. (2004). Reducing false recognition with criterial recollection tests: Distinctiveness heuristic versus criterion shifts. Journal of Memory \& Language, 51, 473-493.

Gellatly, A., Banton, P., \& Woods, C. (1995). Salience and awareness in the Jacoby-Whitehouse effect. Journal of Experimental Psychology: Learning, Memory, \& Cognition, 21, 1374-1379.

Higham, P. A., \& VoKey, J. R. (2000). Judgment heuristics and recognition memory: Prime identification and target-processing fluency. Memory \& Cognition, 28, 575-584.

Jacoby, L. L., Kelley, C. M., \& McElree, B. D. (1999). The role of cognitive control: Early selection versus late correction. In S. Chaiken \& Y. Trope (Eds.), Dual-process theories in social psychology (pp. 383-400). New York: Guilford.

JACOBY, L. L., \& Whitehouse, K. (1989). An illusion of memory: False recognition influenced by unconscious perception. Journal of Experimental Psychology: General, 118, 126-135.

Johnson, M. J., Hashtroudi, S., \& Lindsay, D. S. (1993). Reality monitoring. Psychological Review, 88, 67-85.

Kelley, C. M., \& Rhodes, M. G. (2002). Making sense and nonsense of experience: Attributions in memory and judgments. In B. H. Ross (Ed.), The psychology of learning and motivation (Vol. 41, pp. 293-320). San Diego: Academic Press.

Lloyd, M. E., Westerman, D. L., \& Miller, J. K. (2003). The fluency heuristic in recognition memory: The effect of repetition. Journal of Memory \& Language, 48, 603-614.

RAJARAM, S. (1993). Remembering and knowing: Two means of access to the personal past. Memory \& Cognition, 21, 89-102.

Schacter, D. L., Israel, L., \& Racine, C. (1999). Suppressing false recognition in younger and older adults: The distinctiveness heuristic. Journal of Memory \& Language, 40, 1-24.

Schacter, D. L., \& Wiseman, A. L. (2006). Reducing memory errors: The distinctiveness heuristic. In R. R. Hunt \& J. B. Worthen (Eds.), Distinctiveness and memory (pp. 89-107). Oxford: Oxford University Press.
Snodgrass, J. G., \& VANDERWART, M. (1980). A standardized set of 260 pictures: Norms for name agreement, image agreement, familiarity, and visual complexity. Journal of Experimental Psychology: Human Learning \& Memory, 6, 174-215.

Westerman, D. L. (2001). The role of familiarity in item recognition, associative recognition, and plurality recognition on self-paced and speeded tests. Journal of Experimental Psychology: Learning, Memory, \& Cognition, 27, 723-732.

Westerman, D. L., Lloyd, M. E., \& Miller, J. K. (2002). The attribution of perceptual fluency in recognition memory: The role of expectation. Journal of Memory \& Language, 47, 607-617.

Westerman, D. L., Miller, J. K., \& Lloyd, M. E. (2003). Change in perceptual form attenuates the use of the fluency heuristic in recognition. Memory \& Cognition, 31, 619-629.

Whittlesea, B. W. A. (1993). Illusions of familiarity. Journal of Experimental Psychology: Learning, Memory, \& Cognition, 19, 1235-1253.

\section{NOTES}

1. This interaction was only marginally significant when we excluded responses that occurred outside the response window $[F(1,46)=4.02$, $\left.M S_{\mathrm{e}}=.023, p=.05\right]$, likely because of reduced statistical power.

2 . A reviewer wondered why speeded responding-despite a greater reliance on familiarity-did not enhance the Jacoby-Whitehouse illusion, at least in the auditory condition. However, although we assume that speeded responding decreased recollection-based responding, we do not assume that it would increase the use of familiarity in comparison with the self-paced condition. Because recollection and familiarity were not in opposition in our task, subjects may have relied quite heavily on familiarity, even in the self-paced condition.

(Manuscript received May 15, 2007; revision accepted for publication July 31, 2007.) 